\title{
THE SCHMITTER PROBLEM AND A RELATED PROBLEM: A PARTIAL SOLUTION
}

\author{
BY R. KAAS \\ University of Amsterdam, the Netherlands
}

\begin{abstract}
At the 1990 ASTIN-colloquium, SCHMITTER posed the problem of finding the extreme values of the ultimate ruin probability $\psi(u)$ in a risk process with initial capital $u$, fixed safety margin $\theta$, and mean $\mu$ and variance $\sigma^{2}$ of the individual claims. This note aims to give some more insight into this problem. Schmitter's conjecture that the maximizing individual claims distribution is always diatomic is disproved by a counterexample. It is shown that if one uses the distribution maximizing the upper bound $e^{-R u}$ to find a 'large' ruin probability among risks with range $[0, b]$, incorrect results are found if $b$ is large or $u$ small.

The related problem of finding extreme values of stop-loss premiums for a compound Poisson $(\lambda)$ distribution with identical restrictions on the individual claims is analyzed by the same methods. The results obtained are very similar.
\end{abstract}

\section{INTRODUCTION}

In a paper presented at the ASTIN-colloquium 1990, HANS SCHMITTER gives a derivation of an exact algorithm to compute the value of the ultimate ruin probability $\psi(u)$ for a compound Poisson ruin process with given premium income $c$ per unit of time, and with claims having a finite number of mass points. In connection with this paper, he posed the following problem: given that the individual claims have mean $\mu$ and variance $\sigma^{2}$, which claims distributions minimize and maximize the ruin probability for a given $u$ ? A practical justification of the problem can be found in the paper by BROCKETT, GOOVAERTS and TAYLOR (1991), who also sum up the results of the discussion of this matter at the colloquia of Montreux and subsequently Oberwolfach.

In the classical ruin model, the non-ruin probability of a compound Poisson risk process can be shown to have a compound geometric distribution with geometric parameter depending only on the safety loading $\theta$, and with terms having a distribution function related to the stop-loss premiums of the individual claims.

In this note we also describe another problem, very similar to Schmitter's. Suppose a reinsurer has to determine a stop-loss premium for a risk with the following properties : the risk has a compound Poisson distribution with known 
parameter $\lambda$, and the individual claims have known mean $\mu$ and variance $\sigma^{2}$. To be able to quote a safe premium, the reinsurer tries to determine the claims distribution leading to the maximum value of the net stop-loss premium. Some work in this direction was done by KAAS and GoOvaERTs (1986) and Steenackers and Goovaerts (1990). See also Goovaerts et al. (1984).

A lower bound for both the ruin probability and the compound Poisson stop-loss premium under these restrictions is attained by the distribution concentrating all mass at $\mu$, see for instance GoovaERTs et al. (1990). This distribution is not actually an element of the set of feasible distributions, which is not a closed set. We will prove that both our functionals, ruin probabilities and compound Poisson stop-loss premiums, are continuous at this boundary point. Other functionals, like the variance, the skewness and the adjustment coefficient do not have this property. See Section 2.

In this paper we concentrate on the upper bounds, and indicate how one may find the diatomic claims distribution leading to the highest ruin probability using the algorithm mentioned above. The compound stop-loss premium can be computed by a very similar formula, based on special properties of the compound Poisson distribution. See Section 2. We found counterexamples for Schmitter's conjecture that the maximal ruin probability always is realized by a diatomic distribution. For the compound Poisson stop-loss premiums, the optimal diatomic distribution also was not always the overall maximum. See Section 3.

A useful heuristic approximation to the maximal ruin probability with diatomic claims is described in Section 4. It is based on maximization of the most important term of the geometric distribution. Our limited numerical experience shows that this solution leads to a ruin probability which is invariably close to the maximal diatomic ruin probability. For small $\lambda$, this same diatomic distribution also often leads to near-maximum compound Poisson stop-loss premiums.

One of the referees remarked that applying this heuristic approach one actually solves Schmitter's problem optimally for very small values of the initial capital. More precisely, if the initial capital/the retention is very small (less than $\frac{1}{2} E\left[X^{2}\right] / E[X]$ ), the maximum ruin probability/compound stop-loss premium is attained for the diatomic distribution with 0 as a mass point.

In any case it can be shown that this heuristic solution is better than many other choices of the feasible distribution. If $x_{1}$ and $x_{2}$ are the mass points of the heuristically found feasible distribution, with $x_{1}<x_{2}$, any distribution with least mass point larger than $x_{1}$ leads to lower ruin probabilities and compound Poisson stop-loss premiums.

In Section 5 we impose one more restriction on the claims distribution, namely that the support is contained in an interval $[0, b]$. One might expect that the distribution with the largest value of the upper bound for the ruin probability $e^{-R u}$ also has a high probability of ruin. It can be shown that the adjustment coefficient $R$ with the claims distribution is minimal for the diatomic distribution with $b$ as one of its mass points. Then obviously $e^{-R u}$ is maximal. But if the maximum claim $b$ is very large, the ruin probability with 
this distribution is close to minimal rather than maximal. On the other hand, the adjustment coefficient $R$ is maximal for the diatomic distribution with 0 as a mass point, but for small values of $u$ this distribution has maximal ruin probability, in spite of the fact that is has minimal $e^{-R u}$. So looking at the adjustment coefficient leads to the wrong answer, unless $b$ is small and $u$ is large, say for $b \leq 2 u-\mu$, see the previous paragraph and Section 4 .

In Section 2 it is shown that the third moment (skewness) of the compound Poisson distribution is maximal for the diatomic claims distribution with $b$ as a mass point. So one may expect that for large retentions, this claims distribution leads to maximal stop-loss premiums. Also in Section 5 we will show that for small retentions the situation is reversed.

\section{SOME THEORY AND NOTATION}

In both problems we study, the issue is to find a maximum of a functional $H_{u}$, working on distribution functions $F_{X}$ of random variables $X$ in a certain set. More specifically, we may write both problems in the following form:

(1) Maximize $H_{u}\left[F_{X}\right]$

subject to $\quad X$ is a non-negative random variable, with $E[X]=\mu$, $\operatorname{Var}[X]=\sigma^{2}$.

Here $H_{u}[\cdot]$ assigns to $F_{X}$ either the ruin probability $\psi(u)$ in a compound Poisson risk process with fixed safety loading $\theta$ and initial capital $u$, or the stop-loss premium $\pi_{S}(u)$ at retention $u$ of a compound Poisson $(\lambda)$ distributed random variable $S$, both with individual claims distributed as $X$. In the remainder of this section we will give expressions for $H_{u}[\cdot]$ for both problems in case $X$ has a finite range. Also, we will characterize the feasible random variables $X$ having a two-point support. Finally, the theory of ordering of risks is applied to derive results on some integrals over $H_{u}[\cdot]$.

Consider the classical actuarial ruin model, that is, assume a compound Poisson process with claims intensity $\lambda$, non-negative individual claims distributed as $X$, premium income per unit time $c=(1+\theta) \lambda E[X]$, which means there is a safety loading $\theta$ (assumed positive), and initial capital $u$. See for instance Bowers et al. (1986, Chapter 12). Let the stochastic process $N(t)$ denote the number of claims up to time $t$, and $S(t)=X_{1}+\ldots+X_{N(t)}$ the accumulated claims until time $t$. Define the maximal aggregate loss as $L=$ $\max \{S(t)-c t \mid t \geq 0\}$. The ultimate ruin probability $\psi(u)$ denotes the probability that the insurer's surplus will ever become negative:

$$
\psi(u)=P[\min \{u+c t-S(t) \mid t \geq 0\}<0]=1-P[L \leq u] .
$$

Defining $L_{1}, L_{2}, \ldots$ as the amounts by which record lows in the insurer's surplus $u+c t-S(t)$ are broken, and $M$ to be the number of record lows in the surplus process, we may write 


$$
L=\sum_{i=1}^{M} L_{i}
$$

Then $M$ has a geometric distribution with parameter $\psi(0)$. From Theorem III.2.2.3 in GoovAERTS et al. (1990) we see that the geometric parameter $\psi(0)=(1+\theta)^{-1}$, and the distribution function of the $L_{i}$ equals

$$
F_{L_{i}}(y)=1-\pi_{X}(y) / \pi_{X}(0),
$$

where $\pi_{X}(y)=E\left[(X-y)_{+}\right]$denotes the net stop-loss premium for $X$ at retention $y$, so $\pi_{X}(0)=E[X]$.

From (2) and (3) we obtain the following expression for the ruin probability:

$$
\psi(u)=P[L>u]=\frac{\theta}{1+\theta} \sum_{m=0}^{\infty}\left\{\frac{1}{1+\theta}\right\}^{m} P\left[L_{1}+\ldots+L_{m}>u\right] .
$$

SCHMITTER (1990) gives the following expression for the ruin probability in case $X$ has finite support $\left\{x_{1}, x_{2}, \ldots, x_{m}\right\}$, with associated probabilities $p_{1}, p_{2}, \ldots, p_{m}$ :

$$
\begin{aligned}
& \psi(u)=1-\frac{\theta}{1+\theta} \sum_{k_{1}, k_{2}, \ldots, k_{m}}(-z)^{k_{1}+\ldots+k_{m}} e^{z} \prod_{j=1}^{m} \frac{p_{j}^{k_{j}}}{k_{j} !}, \\
& \text { where } z=\frac{\lambda}{c}\left(u-k_{1} x_{1}-\ldots-k_{m} x_{m}\right)_{+} .
\end{aligned}
$$

Similar expressions can be found in Gerber (1990), SHIU (1989), and earlier TAKÁCs (1967). The indices $k_{j}$ are assumed to range over $0,1, \ldots$ If all mass points $x_{j}$ are strictly positive, $j=1, \ldots, m,(6)$ is a sum with only a finite number of non-zero terms, so it leads to an easily programmed algorithm to compute $\psi(u)$ for discrete claims distributions. If one of the mass points, say $x_{m}$, is equal to 0 , carrying out the (infinite) summation over $k_{m}$ in (6) leads to the same expression as (6) with $m$ replaced by $m-1, \lambda$ by $\lambda\left(1-p_{m}\right)$, and $p_{j}$ by $p_{j} /\left(1-p_{m}\right), j=1, \ldots, m-1$.

In Section III.5 of GoovaerTs et al. (1990) we find that the distributions with mean $\mu$ and variance $\sigma^{2}$ that are diatomic with support $\left\{x_{1}, x_{2}\right\}$, for $x_{1}=\mu-\varepsilon$, can be characterized by

$$
\begin{aligned}
& x_{1}=\mu-\varepsilon, \quad x_{2}=\mu+\sigma^{2} / \varepsilon, \\
& p_{1}=P\left[X=x_{1}\right]=\sigma^{2} /\left\{\sigma^{2}+\varepsilon^{2}\right\}, \quad p_{2}=P\left[X=x_{2}\right]=1-p_{1} .
\end{aligned}
$$

For $0 \leq x_{1}<x_{2}<\infty$, we must have $0 \leq x_{1}<\mu$, so $0<\varepsilon \leq \mu$. Note that $x_{2}$ increases with $x_{1}$ for $x_{1} \in[0, \mu)$.

Inserting (7) in (6) with $m=2$, we see that $\psi(u)$ is continuous for diatomic distributions as a function of $\varepsilon$ at $\varepsilon \downarrow 0$. So there is a sequence of feasible diatomic distributions, whose ruin probabilities converge to the one of the claims distribution with $P[X=\mu]=1$, or $\varepsilon=0$. 
The compound Poisson stop-loss premium can be written in the form

$$
\pi_{S}(u)=\sum_{n=0}^{\infty} \lambda^{n} e^{-\lambda} / n ! E\left[\left(X_{1}+\ldots+X_{n}-u\right)_{+}\right] .
$$

If the range of the claims is finite, there is an expression for the compound stop-loss premiums similar to (6). If $S$ has a compound Poisson $(\lambda)$ distribution with individual claims distribution as in (6), and $N_{j}$ counts the number of occurrences of claim size $x_{j}$, such that $S=x_{1} \cdot N_{1}+\ldots+x_{m} \cdot N_{m}$, then it is well-known that the $N_{j}$ are independent Poisson $\left(\lambda p_{j}\right)$ distributed random variables. So the stop-loss premium of $S$ at retention $u$ can be written as:

(9) $\pi_{S}(u)=E\left[(S-u)_{+}\right]=E[S]-u+E\left[(u-S)_{+}\right]$

$$
=E[S]-u+\sum_{k_{1}, k_{2}, \ldots, k_{m}} e^{-\lambda}\left(u-k_{1} x_{1}-\ldots-k_{m} x_{m}\right)+\prod_{j=1}^{m} \frac{\left(\lambda p_{j}\right)^{k_{j}}}{k_{j} !} .
$$

It is evident that $\pi_{S}(0)=\lambda \mu, \pi_{S}(\infty)=0, \psi(0)=(1+\theta)^{-1}$ and $\psi(\infty)=0$ do not depend on the actual choice of the feasible distribution. We will show that this holds for the integrals over $\pi_{S}(u)$ and $\psi(u)$ as well; the weighted integrals over $u \pi_{S}(u)$ and $u \psi(u)$, however, are minimal/maximal when the third moment of the individual claims is.

We will use the following identities, valid for non-negative random variables $Y$ with $E\left[Y^{j+2}\right]<\infty$, and which can be proved by partial integration:

$$
\begin{aligned}
& \int_{0}^{\infty} y^{j} \pi_{Y}(y) d y=\int_{0}^{\infty} \frac{1}{j+1} y^{j+1}\left[1-F_{Y}(y)\right] d y \\
& \int_{0}^{\infty} y^{j}\left[1-F_{Y}(y)\right] d y=\frac{1}{j+1} E\left[Y^{j+1}\right], \quad j \geq 0 .
\end{aligned}
$$

Using (10) and familiar properties of moments of compound distributions, we may deduce for every feasible distribution of the individual claims:

$$
\begin{aligned}
\int_{0}^{\infty} \pi_{S}(u) d u & =\frac{1}{2} E\left[S^{2}\right]=\frac{1}{2}\left\{\operatorname{Var}[S]+(E[S])^{2}\right\}=\frac{1}{2}\left\{\lambda\left(\sigma^{2}+\mu^{2}\right)+\lambda^{2} \mu^{2}\right\} \\
\int_{0}^{\infty} \psi(u) d u & =E[L]=E[M] E\left[L_{1}\right]=\frac{1}{\theta} \int_{0}^{\infty}\left[1-F_{L_{1}}(u)\right] d u \\
& =\frac{1}{\theta \mu} \int_{0}^{\infty} \pi_{X}(u) d u=\frac{1}{\theta \mu} E\left[\frac{1}{2} X^{2}\right]=\frac{\sigma^{2}+\mu^{2}}{2 \theta \mu}
\end{aligned}
$$


The following relations for weighted integrals hold:

$$
\begin{aligned}
\int_{0}^{\infty} u \pi_{S}(u) d u & =\frac{1}{6} E\left[S^{3}\right]=\frac{1}{6} E\left[(S-E[S]+E[S])^{3}\right] \\
& =\frac{1}{6}\left\{\lambda E\left[X^{3}\right]+3 \lambda^{2} \mu\left(\mu^{2}+\sigma^{2}\right)+\lambda^{3} \mu^{3}\right\} \\
\int_{0}^{\infty} u \psi(u) d u & =\frac{1}{2} E\left[L^{2}\right]=\frac{1}{2} E\left[E\left[L^{2} \mid M\right]\right] \\
& =\frac{1}{2} E\left[M \cdot E\left[L_{1}^{2}\right]+M(M-1)\left(E\left[L_{1}\right]\right)^{2}\right] \\
& =\frac{1}{2} E[M] E\left[L_{1}^{2}\right]+\frac{1}{2} E[M(M-1)]\left(E\left[L_{1}\right]\right)^{2} \\
& =\frac{1}{2 \theta} E\left[L_{1}^{2}\right]+\frac{1}{\theta^{2}}\left(E\left[L_{1}\right]\right)^{2}=\frac{1}{6 \theta \mu} E\left[X^{3}\right]+\frac{\left(\sigma^{2}+\mu^{2}\right)^{2}}{4 \theta^{2} \mu^{2}} .
\end{aligned}
$$

So the fatter the tail of the individual claims $X$ (measured by their skewness, or what is the same since $\mu$ and $\sigma^{2}$ are given, by their third moment), the larger the integral over $u \psi(u)$ and $u \pi_{S}(u)$.

In the theory of ordering of risks as described in GoovaerTs et al. (1990), one compares stop-loss transforms or distribution functions of risks over the whole interval $[0, \infty)$. In our case it is sufficient if these functions are ordered only on the interval $[0, u]$. Suppose that for instance $X$ has lower stop-loss premiums than $Y$ on the interval $[0, u]$. If $Z$ is another independent risk, we have

$$
\begin{aligned}
E\left[(X+Z-u)_{+}\right]= & \int_{0}^{\infty} E\left[(X+Z-u)_{+} \mid Z=z\right] d F_{Z}(z) \\
= & \int_{0}^{\infty} E\left[(X-(u-z))_{+}\right] d F_{Z}(z) \\
& \leq \int_{0}^{\infty} E\left[(Y-(u-z))_{+}\right] d F_{Z}(z)=E\left[(Y+Z-u)_{+}\right]
\end{aligned}
$$

From this porperty we see directly that if $X_{1}, X_{2}, \ldots$ and $Y_{1}, Y_{2}, \ldots$ are sequences of independent risks distributed as $X$ and $Y$ respectively, and $X$ has lower stop-loss premiums than $Y$ on $[0, u]$, then we have $E\left[\left(X_{1}+\ldots+X_{m}-u\right)_{+}\right] \leq E\left[\left(Y_{1}+\ldots+Y_{m}-u\right)_{+}\right]$for all $m=1,2, \ldots$ Using (8), we see that a compound Poisson distribution with $X$ as claims distribution has a lower stop-loss premium in $u$ than one with $Y$. Using (4) and (5), we see that ruin probabilities are lower as well.

\section{MAXIMIZING THE FUNCTIONALS NUMERICALLY}

It is easy to maximize the ruin probability numerically over the diatomic feasible distributions. This can be accomplished using algorithm (6), together with (7) to characterize the feasible diatomic distributions. It involves merely a 
one-dimensional maximization over the interval $x_{1} \in[0, \mu]$. To do this, one first computes (6) at a number of values of $x_{1}$ to detect the interval in which the maximum is to be found, and subsequently uses a method like golden section search to determine the maximum more exactly. A reference for numerical techniques to compute a maximum of a function over an interval is Press et al. (1986). In Figure 1 we give graphs depicting the diatomic ruin probability $\psi\left(u ; x_{1}, x_{2}, p_{1}, p_{2}\right)=\psi\left(u ; x_{1}\right)$ as a function of $x_{1} \in[0, \mu]$, where $x_{1}, x_{2}, p_{1}, \mathrm{p}_{2}$ are related by (7). We took $\mu=3, \sigma^{2}=1, \theta=0.5$, and $u=1.5,4.5$ and 9 respectively. In these graphs, the scale in the $y$-direction varies.

As announced, the ruin probability is minimal and continuous at $x_{1} \uparrow \mu$. In Figure 1 we see that for small $u\left(u=1 \frac{1}{2}\right)$ the maximum ruin probability is found taking $x_{1}=0$. A close inspection reveals that the ruin probability does not depend on $x_{1}$ if $x_{1} \geq u$. Indeed in (6) one sees that the ruin probability does not (directly) depend on mass points larger than $u$. It also follows from (4) and (5). For large $u(u=9), \psi(u)$ is very nearly constant for small to moderate values of $x_{1}$, then increases, and next decreases steeply to its minimal value at $x_{1} \uparrow \mu$.

For intermediate $u(u=4.5)$, the situation is rather unclear: there are some local maxima. For this specific situation we were able to find a three-point distribution with a larger ruin probability than the one corresponding to the maximizing diatomic distribution. In fact, for

$$
\begin{aligned}
& x_{1}=1.56592, \quad x_{2}=2.67226, \quad x_{3}=5.182086, \\
& p_{1}=0.071198, \quad p_{2}=0.766835, \quad p_{3}=0.161967
\end{aligned}
$$

the ruin probability is 0.279271 , which, although (probably) not the optimal solution, is higher than the maximal diatomic ruin probability 0.279185 , found at $x_{1}=2.5597, x_{2}=5.2712$.

Although we tried a lot of combinations of $\mu, \sigma^{2}, \theta$ and $u$, we rarely found a randomly generated three-point distribution better than the best diatomic distribution; if we did, the difference was never substantial.

We did not try to optimize systematically over all three-point spectra. First, this is not a trivial task : if the number of mass points is $m$, the number of free variables equals $2 m-3$, being the number of support points $x_{j}$ plus the number of probabilities $p_{j}$, minus the number of restrictions. So to find the maximal ruin probability over all three-point spectra involves solving a threedimensional maximization, with borderline conditions $p_{j} \geq 0$. Second, even supposing we successfully optimized over three-point distributions, there is still no guarantee that for instance a 15-point support might not be better.

The fact that for small $u$ the ruin probability is maximal at $x_{1}=0$ can be explained as follows. By relation (11), one sees that neither $\psi(0)$ and $\psi(\infty)$, nor $\int \psi(u) d u$ depend on $x_{1}$. By (12), however, we see that the weighted integral increases (linearly) with the third moment of the claims distribution. So the weighted integral is minimal for the diatomic distribution with $x_{1}=0$, which means that taking $x_{1}=0$ gives the smallest integral over $u \psi(u)$. So at small values of $u, \psi(u)$ should be large for $x_{1}=0$. By similar reasoning, one 

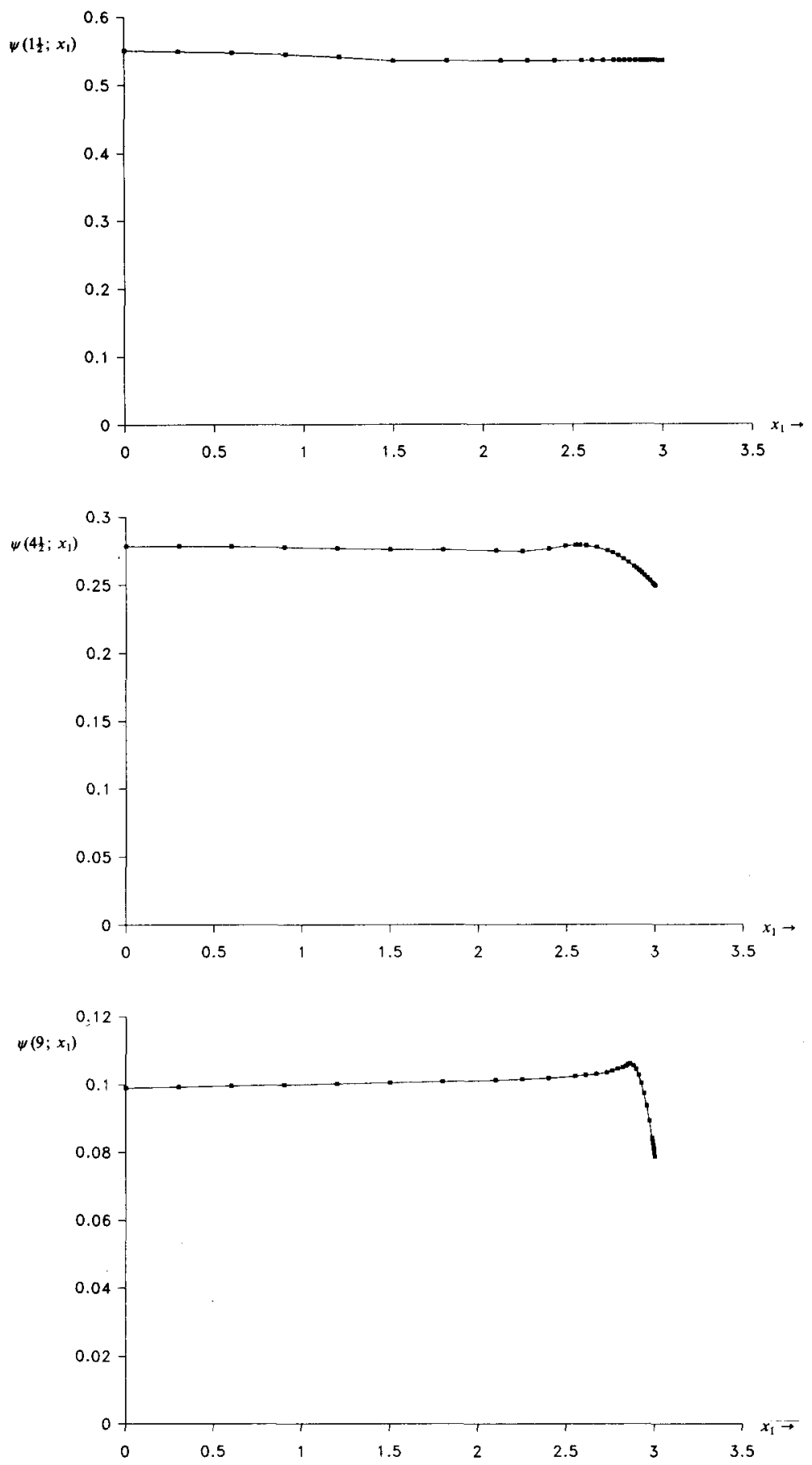

FIGURE 1. $\psi\left(u ; x_{1}\right)$ as a function of $x_{1}, \mu=3, \sigma^{2}=1, \theta=\frac{1}{2} ; u=1 \frac{1}{2}, 4 \frac{1}{2}, 9$. 
explains that for large $u$, a large value of $x_{1}$ leads to maximum $\psi(u)$. For too large values of $x_{1}$, we obtain low ruin probabilities (close to the minimal value), as explained in the following section.

For the same reasons, one can expect a similar pattern to arise in the case of compound Poisson stop-loss premiums. This is indeed the case: see Figure 2. In this figure, we took $\lambda=2, \mu=3$ and $\sigma^{2}=1$. At small $u(u=2)$, the stop-loss premium is virtually constant over $x_{1}$, but it is maximal at $x_{1}=0$. At large $u=20$, we see that the stop-loss premium is practically constant for $x_{1}$ from 0 (where it equals 0.0109 ) to very close to $\mu$. Then it increases very steeply to its maximum value 0.0522 , and for $x_{1} \uparrow \mu$, it decreases continuously to its minimal value of 0.0088 . For intermediate $u=7$, with increasing $x_{1}, \pi_{S}(u)$ increases slightly and irregularly at first from 1.3373 to the maximal value 1.3954, and then for $x_{1} \uparrow \mu$, it decreases again to its infimum 1.3008. For this case we found again an example where the maximal diatomic distribution was not a global maximum over all feasible claims distributions. The maximal diatomic distribution is at $x_{1}=2 \frac{2}{3}$, where $\pi_{S}(u)=1.3954$, but a larger stop-loss premium of 1.3995 is attained by the triatomic distribution

$$
x_{1}=0, x_{2}=2.8, x_{3}=5.7143, p_{1}=0.0286, p_{2}=0.8754, p_{3}=0.0961 \text {. }
$$

In fact, as one of the referees pointed out, it can be proven that the diatomic distribution with $x_{1}=0$ as a mass point is optimal for very small values of $u$ $\left(u \leq \frac{1}{2} E\left[X^{2}\right] / E[X]\right)$. The proof goes as follows.

From Theorem III.5.2.3 of GoOvaERTS et al. (1990) we see that uniformly for all $u \leq \frac{1}{2} E\left[X^{2}\right] / E[X]=\frac{1}{2}\left(\mu+\sigma^{2} / \mu\right)$, the maximal stop-loss premium over the feasible distributions is attained for a random variable $X_{0}$ having mass points 0 and $\mu+\sigma^{2} / \mu$, see (7). As a consequence of (13), we have immediately that if $H$ is the distribution function of $X_{0}$ and $X$ is a feasible claim size, then $F_{X}^{* n}$ has smaller stop-loss premium in $u$ than $H^{* n}$ for $n=2,3, \ldots$, too. In view of (8), we have then found that $H$ is the claims distribution maximizing the compound Poisson stop-loss premium, when the retention $u \leq \frac{1}{2}\left(\mu+\sigma^{2} / \mu\right)$.

Using (4), we can deduce by similar reasoning that this same claims distribution also maximizes not only $P\left[L_{1}>u\right]$ for $u \leq \frac{1}{2}\left(\mu+\sigma^{2} / \mu\right)$, but also $P\left[L_{1}+\ldots+L_{m}>u\right]$ for all $m=2,3, \ldots$, and thus maximizes the ruin probability (5).

So Schmitter's problem is solved for very small values of the initial capital $u$.

This result is confirmed in Figure 1 for $u=1 \frac{1}{2}$. But note that in Figure 2 for $u=2>\frac{1}{2}\left(\mu+\sigma^{2} / \mu\right)$ still the distribution having mass point 0 led to the maximal compound Poisson stop-loss premium.

\section{AN APPROXIMATION FOR THE MAXIMIZING DIATOMIC DISTRIBUTION}

Though we are as yet unable to solve the problem of maximizing $\psi(u)=P[L>u]$ given $\mu$ and $\sigma^{2}$, a problem we can solve is the maximization of $P\left[L_{1}>u\right]$. We may expect $P[L>u]$ to be large when $P\left[L_{1}>u\right]$ is, because the term with $m=1$ in (5) has the largest weight factor. 

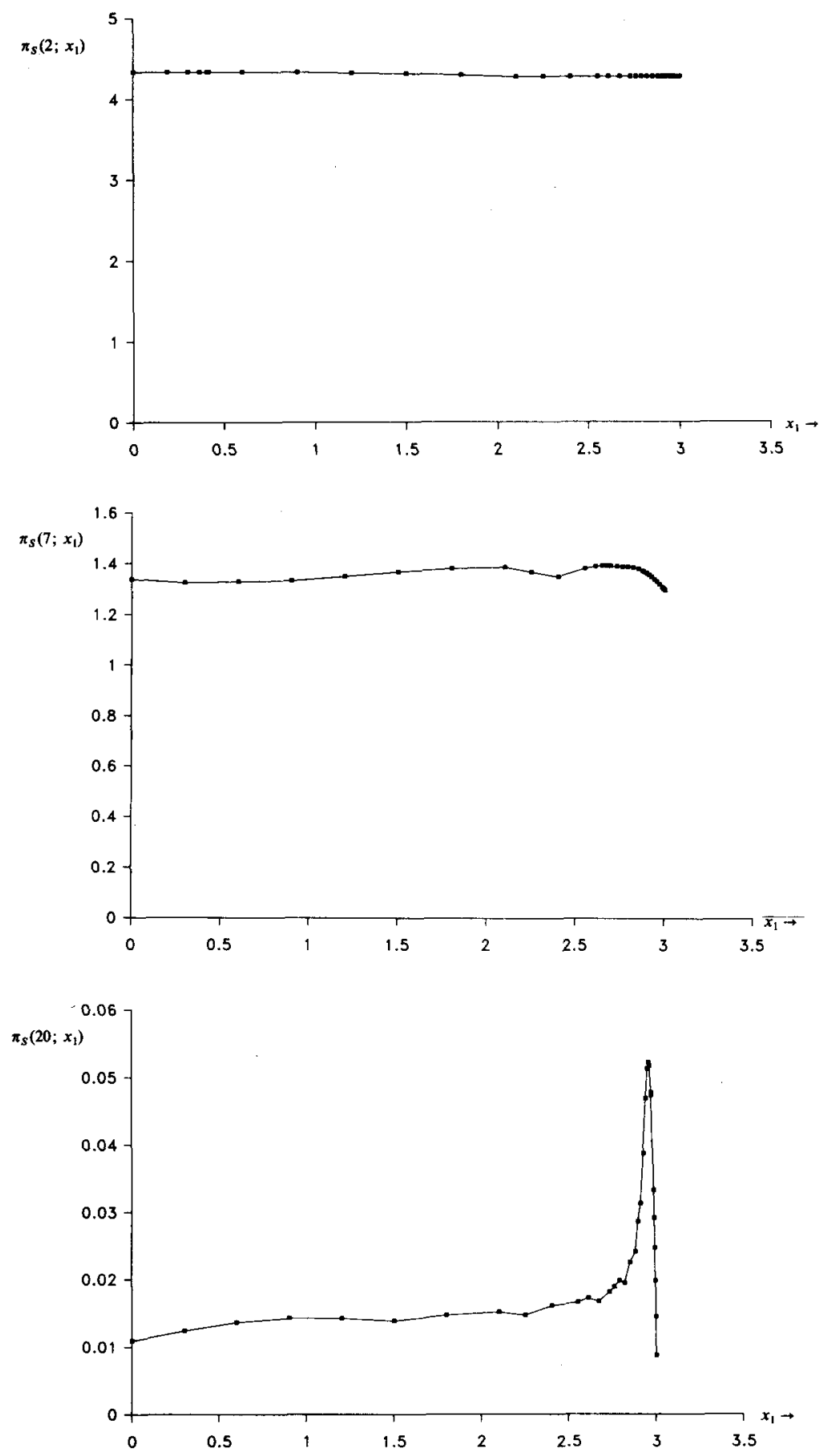

FIGURE 2. $\pi_{S}\left(u ; x_{1}\right)$ as a function of $x_{1}, \mu=3, \sigma^{2}=1, \theta=\frac{1}{2} ; u=2,7,20$. 
In view of (4), and since $\pi_{X}(0)=E[X]=\mu$ is given, to maximize $P\left[L_{1}>u\right]$ we just have to maximize $\pi_{X}(u)$, the stop-loss premium of $X$. The solution to this problem can for instance be found in GoovaerTs et al. (1990), Theorems III.5.2.2 and 5.2.3. These theorems express that the maximal stop-loss premium for a (non-negative) risk $X$ with mean $\mu$ and variance $\sigma^{2}$ at retention $u$ is the diatomic distribution with smaller mass point $x_{1}=\max \{u-d, 0\}$, where $d=\left\{(\mu-u)^{2}+\sigma^{2}\right\}^{\frac{1}{2}}$. When $\sigma$ is small with respect to $|u-\mu|$, we may write

$$
(u-\mu)-d=(u-\mu-d) \frac{u-\mu+d}{u-\mu+d}=\frac{-\sigma^{2}}{u-\mu+d} \approx-\frac{1}{2} \sigma^{2} /(u-\mu)
$$

So we may conclude that the diatomic distribution with the following mass points gives a 'high' ruin probability:

$$
x_{1}=\mu-\varepsilon, \text { with } \varepsilon=\frac{\sigma^{2}}{u-\mu+d} \approx \frac{1}{2} \sigma^{2} /(u-\mu), \text { so } x_{2}=u+d \approx 2 u-\mu .
$$

In the examples we tested, the diatomic distribution maximizing the ruin probability had $x_{1}$ only slightly smaller than $u-d$. See Table 1 .

Of course this same diatomic distribution maximizes the term with $n=1$ of the compound Poisson stop-loss premium (8). So one may expect this distribution to have a high stop-loss premium if the probability of just one claim is large, which is the case if $\lambda$ is small. For large $\lambda$, however, this approximation will not be as useful.

Our heuristic procedure may not always lead to the optimal value, but it can be shown that it is better than many other choices. Suppose $Z$ has distribution (15), and suppose $Y$ is another feasible choice such that the least mass point of $Y$ is larger than that of $Z$, which is $u-d$. We know that $\pi_{Z}(t)$ is piecewise linear, with edges at $u-d$ and $u+d$. Since $Y$ has no mass below $u-d$, we have $\pi_{Y}(u-d)=\pi_{Z}(u-d)$. Also, $\pi_{Y}(u) \leq \pi_{Z}(u)$ since $\pi_{Z}(u)$ is maximal. So $\pi_{Y}(t) \leq \pi_{Z}(t)$ for all $t \leq u$, which means that $Y$ generates lower compound Poisson stop-loss premiums and ruin probabilities.

TABLE 1

\begin{tabular}{|c|c|c|c|c|c|c|}
\hline \multirow[b]{2}{*}{$x_{2}=$} & \multicolumn{3}{|c|}{$\mu=1, \sigma^{2}=1, \theta=1$} & \multicolumn{3}{|c|}{$\mu=3, \sigma^{2}=1, \theta=.5$} \\
\hline & $\begin{array}{l}u=1.5 \\
\psi(u)=\end{array}$ & $\begin{array}{l}u=4.5 \\
\psi(u)=\end{array}$ & $\begin{array}{c}u=9 \\
\psi(u)=\end{array}$ & $\begin{array}{l}u=1.5 \\
\psi(u)=\end{array}$ & $\begin{array}{l}u=4.5 \\
\psi(u)=\end{array}$ & $\begin{array}{c}u=9 \\
\psi(u)=\end{array}$ \\
\hline$\infty$ & .102003 & .002315 & .000008 & .534796 & .248974 & .078779 \\
\hline$\mu+\sigma^{2} / \mu$ & .272504 & .039292 & .002315 & .550047 & .278350 & .098945 \\
\hline optimal & .275023 & .081105 & .034151 & .550047 & .279190 & .106205 \\
\hline$u+d$ & .269824 & .078214 & .033632 & .534796 & .276506 & .101811 \\
\hline $2 u-\mu$ & .272504 & .078651 & .033659 & .550047 & .277596 & .101901 \\
\hline 10 & .146348 & .071460 & .024767 & .534796 & .265714 & .106184 \\
\hline 15 & .130637 & .055095 & .034151 & .534796 & .259498 & .101901 \\
\hline 20 & .123125 & .044244 & .031936 & .534796 & .256613 & .097203 \\
\hline
\end{tabular}

VALUES OF $\psi(u)$ FOR DIFFERENT VALUES OF THE HIGHER MASS POINT IN A DIATOMIC DISTRIBUTION 
In particular, the diatomic solutions with support $\left\{b, b^{\prime}\right\}$ with $b>u-d$ are apparently non-optimal.

\section{EXTREMAL VALUES OF THE ADJUSTMENT COEFFICIENT}

Consider all claims distributions with mean $\mu$, variance $\sigma^{2}$ and as an extra requirement, support contained in $[0, b]$ for some $b \geq \mu+\sigma^{2} / \mu$. Just as we did in the previous section for $P\left[L_{1}>u\right]$, one may tackle the problem of finding extremal ruin probabilities by using distributions leading to extremal values of related quantities like an approximation or an upper bound for the ruin probability. Here we use the upper bound $e^{-R u}$, where the adjustment coefficient $R$ is the positive solution to the equation

$$
1+(1+\theta) \mu r=E\left[e^{r X}\right] \text {. }
$$

Asymptotically, this upper bound can be used as an approximaton, since $\psi(u) e^{R u}$ has a limit in $(0,1)$ for $u \rightarrow \infty$.

It can easily be shown that the diatomic distribution with mass points 0 and $\mu+\sigma^{2} / \mu$ is minimal in second degree stop-loss order, while the one with mass points $b$ and $\mu-\sigma^{2} /(b-\mu)$ is maximal. See Theorem II.4.2.3 of GoovaERTs et al. (1990). This implies that these special diatomic distributions have minimal and maximal moment generating functions on $(0, \infty)$ in the class considered, and accordingly the corresponding adjustment coefficients (roots of (16)) are maximal and minimal respectively.

One would expect that the support $\left\{\mu-\sigma^{2} /(b-\mu), b\right\}$, with minimal adjustment coefficient, leads to large ruin probability, too. Taking $b$ too large, however, so $\mu-\sigma^{2} /(b-\mu)$ is very close to $\mu$, results in the opposite of what we wanted: the ruin probability of this distribution is very small rather than maximal. For $b \rightarrow \infty$, by (7) we see that the $m g f E\left[e^{r X}\right] \rightarrow \infty$ for all $r>0$, so then $R \rightarrow 0$, which gives us the trivial upper bound $\psi(u) \leq 1$. So we observe that for $b \rightarrow \infty$, the upper bound $e^{-R u}$ increases, while the ruin probability decreases. But if $b$ is not too large, say such that $\mu-\sigma^{2} /(b-\mu) \approx x_{1}$ as in (15), which means that $b \approx 2 u-\mu$, this distribution does lead to a large ruin probability.

On the other hand we learn for instance from Figure 1 that for small $u$, the diatomic distribution with mass point $x_{1}=0$ has maximal ruin probability, even though it gives the tightest upper bound $e^{-R u}$.

It can be shown, too, that the compound Poisson distributions with these distributions for the individual claims are extremal in second degree stop-loss order. This means that they have minimal and maximal third moment, and since mean and standard deviation are fixed, also minimal and maximal coefficient of skewness. As proved at the end of Section 2, these same special spectra also generate the extreme values of $\int u \pi_{S}(u) d u$. So one would be inclined to expect that they lead to. high and low values of the compound Poisson stop-loss premium as well, but the same caveats as above apply here. 


\section{SOME FINAL REMARKS}

To conclude, we comment on tables of some results for distributions with support $\left\{\mu-\sigma^{2} /(b-\mu), b\right\}$ for different values of $b$. These distributions have minimal adjustment coefficient (maximal skewness) for all feasible distributions with support contained in $[0, b]$. They are compared to other distributions described above: the optimal diatomic distribution, the heuristical approximations to the optimum found by applying (15) and the distributions with only one positive mass point: support $\left\{0, \mu+\sigma^{2} / \mu\right\}$ and $\{\mu\}$. The latter support is denoted by higher mass point $\infty$, where the mass on $\infty$ is of course 0 (but contributes to $\sigma^{2}$ ). Note that for $u$ not too large and $b=20$, the phenomenon described above indeed occurs. Even though we showed that looking at the minimal adjustment coefficient sometimes gives incorrect results, especially for large $b$ or small $u$, we fear that this method will be used quite often.

Further note that for large $u$ and $\sigma^{2}$, minimal and maximal ruin probability are widely apart. For $\sigma^{2}$ small with respect to $u$ and $\mu$, the ruin probability cannot vary enormously.

Table 2 gives some results for the compound Poisson stop-loss premiums. Note the meaningless results obtained by the wrong choice of $b$ for large values of $u$, and also for small values of $u$.

An approach that we plan to follow in the near future is to try to optimize the compound Poisson stop-loss premium over the set of claim distributions with support $\{0, \delta, 2 \delta, \ldots, n \delta\}$. The more general problem is obtained taking limits for $n \rightarrow \infty$ and $\delta \downarrow 0$. The restricted problem can be written in the form of the maximization of a non-linear criterion function with three linear constraints on the probabilities $p_{j}=P[X=j \delta]$, required to be non-negative

TABLE 2

VALUES OF $\pi_{S}(u)$ FOR DIFFERENT VALUES OF THE HIGHER MASS POINT IN A DIATOMIC DISTRIBUTION

\begin{tabular}{|c|c|c|c|c|c|c|}
\hline \multirow[b]{2}{*}{$x_{2}=$} & \multicolumn{3}{|c|}{$\mu=3, \sigma^{2}=1, \lambda=2$} & \multicolumn{3}{|c|}{$\mu=3, \sigma^{2}=1, \lambda=5$} \\
\hline & $\begin{array}{c}u=2 \\
\pi_{S}(u)=\end{array}$ & $\begin{array}{c}u=7 \\
\pi_{S}(u)=\end{array}$ & $\begin{array}{c}u=20 \\
\pi_{S}(u)=\end{array}$ & $\begin{array}{c}u=5 \\
\pi_{S}(u)=\end{array}$ & $\begin{array}{c}u=20 \\
\pi_{S}(u)=\end{array}$ & $\begin{array}{c}u=40 \\
\pi_{S}(u)=\end{array}$ \\
\hline$\infty$ & 4.270671 & 1.300816 & 0.008804 & 10.101076 & 1.004413 & 0.002488 \\
\hline$\mu+\sigma^{2} / \mu$ & 4.330598 & 1.337326 & 0.010879 & 10.138862 & 1.077055 & 0.003859 \\
\hline optimal & 4.332192 & 1.395435 & 0.052178 & 10.138862 & 1.136463 & 0.058680 \\
\hline$u+d$ & 4.331675 & 1.374006 & 0.047330 & 10.105046 & 1.077758 & 0.049633 \\
\hline $2 u-\mu$ & 4.324805 & 1.374694 & 0.047347 & 10.105033 & 1.077807 & 0.049638 \\
\hline 5 & 4.270671 & 1.376488 & 0.014677 & 10.101069 & 1.105061 & 0.005110 \\
\hline 10 & 4.270671 & 1.380493 & 0.022903 & 10.104438 & 1.113764 & 0.007883 \\
\hline 15 & 4.270671 & 1.356405 & 0.034962 & 10.103393 & 1.124541 & 0.012330 \\
\hline 20 & 4.270671 & 1.342594 & 0.047335 & 10.102812 & 1.116290 & 0.018726 \\
\hline 25 & 4.270671 & 1.334135 & 0.052137 & 10.102458 & 1.103217 & 0.028545 \\
\hline 30 & 4.270671 & 1.328482 & 0.051061 & 10.102223 & 1.091199 & 0.040868 \\
\hline
\end{tabular}


for all $j$. By restricting to an arithmetic spectrum we are able to use Panjer's recursion instead of (9); the necessary partial derivatives can also be computed by a recursive scheme. The procedure can be generalized if more moments are known.

Of course, as the title of our paper indicates, maximization over the diatomic distributions only does not give a complete solution of either problem. We find, however, that by using this technique both problems are sufficiently solved for practical purposes. In the first place, our examples led us to the conviction that, although the optimal diatomic distribution is not always globally optimal, it is not much removed from this optimum. Second, in our opinion in practice one might judge the attractiveness of risks or risk processes with known mean and variance of the claims by the worst feasible diatomic distribution as well as by the overall worst feasible distribution.

\section{ACKNOWLEDGMENT}

The author wishes to thank ANGEla van HEERWAARDEN for some constructive suggestions, and MARC GoOVAERTS for stimulating discussions. Also, the valuable contributions of both referees are acknowledged.

\section{REFERENCES}

Bowers, N. L., Gerber, H.U., Hickman, J.C., Jones, D.A. and Nesbitt, C.J. (1986) Actuarial Mathematics. Society of Actuaries, Itasca, Illinois.

Brockett, P.L., Goovaerts, M.J. and TAYLOR, G.C. (1991) The Schmitter problem. ASTIN Bulletin 21, 129-132.

Gerber, H.U. (1990) From the convolution of uniform distributions to the probability of ruin. Mitteilungen der VSVM Heft 2/1989, 283-292.

Goovaerts, M.J., De Vylder, F. and Haezendonck, J. (1984) Insurance premiums. NorthHolland, Amsterdam.

Goovaerts, M.J., KaAs, R., Van Heerwaarden, A.E. and Bauwelinckx, T. (1990) Effective Actuarial Methods. North-Holland, Amsterdam.

KAAs, R. and Goovaerts, M.J. (1986) Bounds on stop-loss premiums for compound distributions. ASTIN Bulletin XVI, 1, 13-17.

Press, W.H., Flannery, B.P., Teukolsky, S.A. and Vetterling, W.A. (1986) Numerical recipes; the art of scientific computing. Cambridge University Press.

SCHMITTER, H. (1990) The ruin probability of a discrete claims distribution with a finite number of steps. Paper presented at the XXII ASTIN-colloquium, Montreux, Switzerland.

SHIU, E.S.W. (1989) Ruin probability by operational calculus. Insurance: Mathematics and Economics 8, 243-249.

STEENACKerS, A. and Goovaerts, M.J. (1990) Bounds on stop-loss premiums and ruin probabilities for given values of the mean, variance and maximal value of the claim size. Paper presented at the XXII ASTIN-colloquium, Montreux, Switzerland.

TAKÁCS, L. (1967) Combinatorial Methods in the Theory of Stochastic Processes. Wiley, New York, Reprinted by Krieger, Huntington, NY (1977).

\section{RoB KaAS}

Institute for Actuarial Science and Econometrics, University of Amsterdam, Jodenbreestraat 23, NL-1011 NH Amsterdam, the Netherlands. 